Research Article

\title{
Finite Element Analysis of a Novel Anterior Locking Plate for Thoracolumbar Burst Fracture
}

\author{
Pengcheng Ren $\left(\mathbb{D},{ }^{1}\right.$ Xiaodong Cheng, ${ }^{2}$ Chongyao Lu, ${ }^{1}$ Haotian Wu, ${ }^{1}$ Shuangquan Yao, \\ Sidong Yang $\mathbb{D},{ }^{1,3}$ and Zhaohui Song $\mathbb{D}^{1}$ \\ ${ }^{1}$ Department of Orthopedic Surgery, The Third Hospital of Hebei Medical University, No. 139 Ziqiang Road, Qiaoxi District, \\ Shijiazhuang 050051, China \\ ${ }^{2}$ Key Laboratory of Biomechanics of Hebei Province, Shijiazhuang, Hebei, China \\ ${ }^{3}$ Australian Institute for Bioengineering and Nanotechnology, The University of Queensland, Australia
}

Correspondence should be addressed to Zhaohui Song; bxyszh@yeah.net

Received 31 August 2021; Accepted 29 September 2021; Published 11 October 2021

Academic Editor: Pei Li

Copyright (c) 2021 Pengcheng Ren et al. This is an open access article distributed under the Creative Commons Attribution License, which permits unrestricted use, distribution, and reproduction in any medium, provided the original work is properly cited.

\begin{abstract}
Purpose. The finite element analysis method was used to explore the biomechanical stability of a novel locking plate for thoracolumbar burst fracture fusion fixation. Methods. The thoracolumbar CT imaging data from a normal volunteer was imported into finite software to build a normal model and three different simulated surgical models (the traditional doublesegment fixation model $\mathrm{A}$, the novel double-segment fixation model $\mathrm{B}$, and the novel single-segment fixation model $\mathrm{C}$ ). An axial pressure $(500 \mathrm{~N})$ and a torque $(10 \mathrm{Nm})$ were exerted on the end plate of T12 to simulate activity of the spine. We recorded the range of motion (ROM) and the maximum stress value of the simulated cages and internal fixations. Results. Model A has a larger ROM in all directions than model B (flexion 5.63\%, extension $38.21 \%$, left rotation $46.51 \%$, right rotation $39.76 \%$, left bending $9.45 \%$, and right bending $11.45 \%$ ). Model C also has a larger ROM in all directions than model B (flexion $555.63 \%$, extension $51.42 \%$, left rotation $56.98 \%$, right rotation $55.42 \%$, left bending $65.67 \%$, and right bending $59.47 \%$ ). The maximum stress of the cage in model A is smaller than that in model B except for the extension direction (flexion $96.81 \%$, left rotation $175.96 \%$, right rotation $265.73 \%$, left bending $73.73 \%$, and right bending $171.28 \%$ ). The maximum stress value of the internal fixation in model A is greater than that in model B when models move in flexion (20.23\%), extension (117.43\%), and left rotation $(21.34 \%)$. Conclusion. The novel locking plate has a smaller structure and better performance in biomechanical stability, which may be more compatible with minimally invasive spinal tubular technology.
\end{abstract}

\section{Introduction}

Traditional anterior surgery is recommended for patients with nerve compression from the front, the intact posterior ligamentous complex (PLC), and incomplete spinal cord injury [1-3]. This procedure can perform the decompression and fusion operation of the anterior middle column under direct vision to provide a better nerve decompression effect and fusion stability [4]. However, it is full of controversy due to the complicated surgical approach and many postoperative complications [5-8]. With the development of spine devices, anterior minimally invasive spinal tubular technology, such as anterior lumbar interbody fusion (ALIF), obli- que lateral interbody fusion (OLIF), and direct lateral interbody fusion (DLIF), which requires smaller incisions and avoids excessive approach-related injury while allowing rapid recovery $[9,10]$, has become more popular in recent years. This technique is mostly used in the treatment of intervertebral discs, but is rarely used in the treatment of vertebral body fusion for spinal fractures [11-13].

In the course of clinical treatment, we try to use minimally invasive tubular technology to treat the thoracolumbar burst fractures that require anterior decompression and fusion. However, the traditional anterior fixation instrument cannot well cooperate with the channel technology to perform the operation. Based on this feature, we designed and 


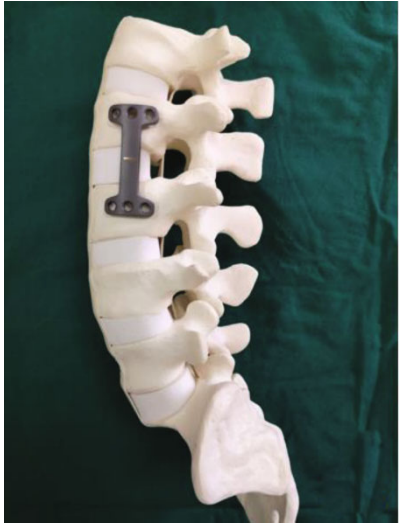

(a)

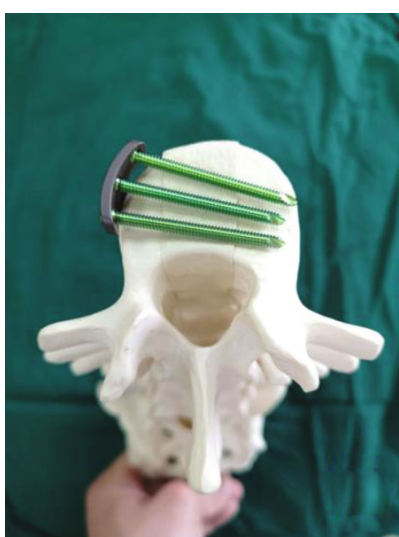

(b)

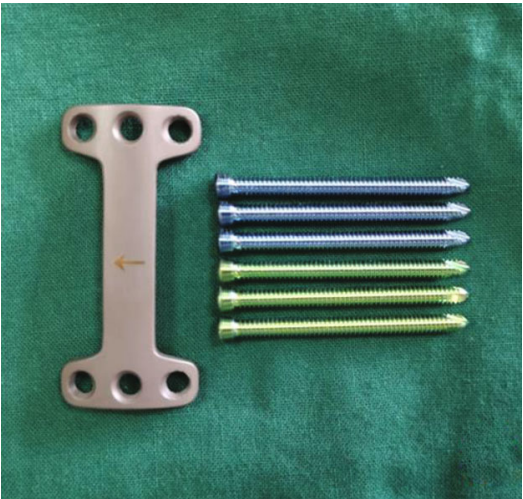

(c)

Figure 1: Preliminary design of the novel plate appearance. (a) The position of the plate on the spine mold. (b) Screws position under transverse section. (c) The shape of the novel plate and screws.

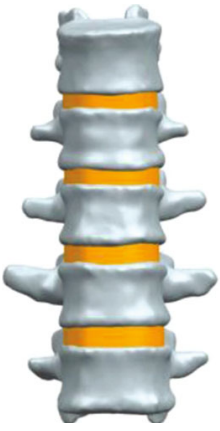

(a)

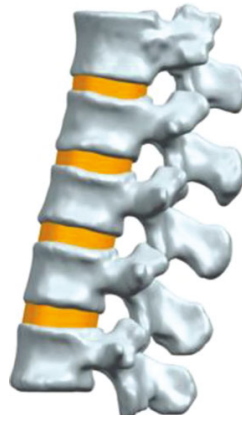

(b)

FIgURE 2: The normal T12-L4 thoracolumbar model designed in UG NX software. (a) Normal model anteroposterior view. (b) Normal model lateral view.

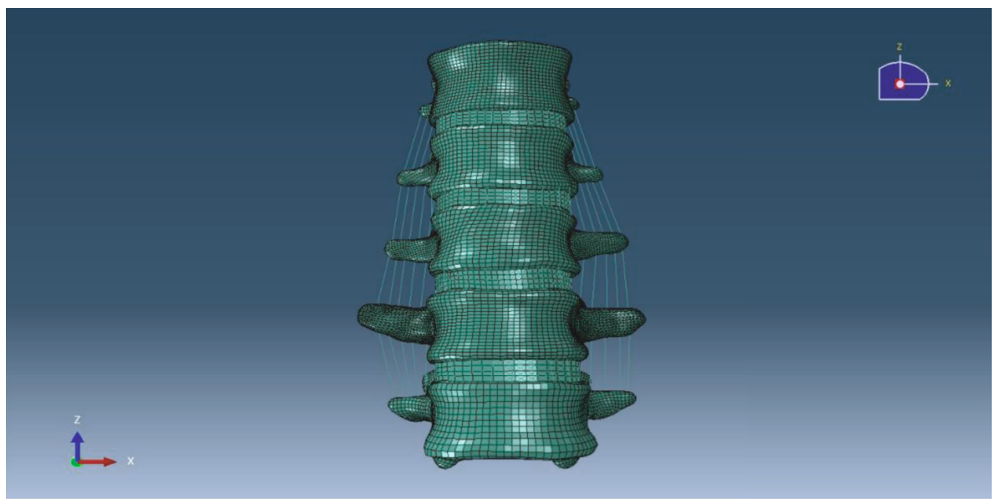

Figure 3: The normal T12-L4 thoracolumbar model (M0) in finite element software Abaqus. Imported the meshed 3D model into the finite element software, build ligaments model, and assigned material properties.

invented an internal fixation device (Figure 1) (Patent No., ZL 201810805552.8) that can meet the requirements of a smaller size, a more concise connection method, and provide better stability.

In order to evaluate the biomechanical properties of the novel plate, this experiment uses finite element analysis to compare the novel fixation instrument with the traditional double-segment fixation instrument (TDFI). By analyzing statistics of the range of motion (ROM) and the maximum stress value of the simulated cages and internal fixations, we try to appraise the biomechanical stability of the novel instrument in single-segment fixation instrument (NSFI) and double-segment fixation instrument (NDFI).

\section{Materials and Methods}

2.1. Novel Plate Design. The appearance of the new plate we designed is shown in Figure 1. The material of the new 
TABLE 1: Material properties of the finite element models.

\begin{tabular}{|c|c|c|c|}
\hline Structures & Elastic modulus (MPa) & Poisson ratio & Sectional area $\left(\mathrm{mm}^{2}\right)$ \\
\hline Cortical bone & 12000 & 0.3 & \\
\hline Cancellous bone & 100 & 0.3 & \\
\hline Annular fiber & 450 & 0.45 & \\
\hline Nucleus pulposus & 1 & 0.49 & \\
\hline Anterior longitudinal ligament & 7.8 & 0.3 & 49 \\
\hline Posterior longitudinal ligament & 10 & 0.3 & 30 \\
\hline Ligamentum flavum & 15 & 0.3 & 40 \\
\hline Interspinous ligament & 10 & 0.3 & 70 \\
\hline Supraspinous ligament & 10 & 0.3 & 70 \\
\hline Intertransverse ligament & 10 & 0.3 & 2 \\
\hline Internal fixation devices & 110000 & 0.3 & \\
\hline
\end{tabular}

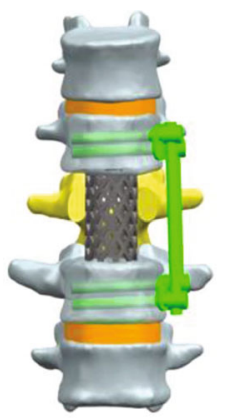

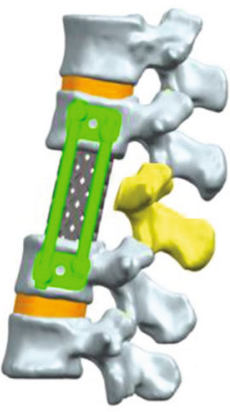

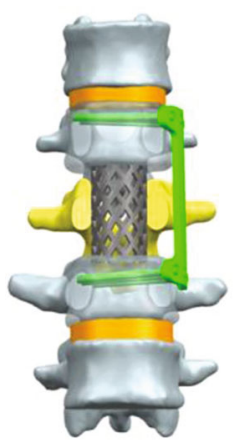

(a)

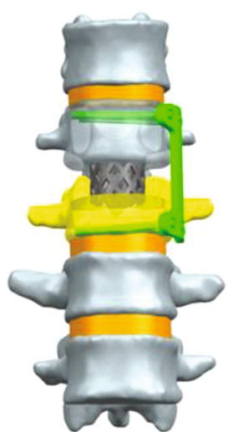

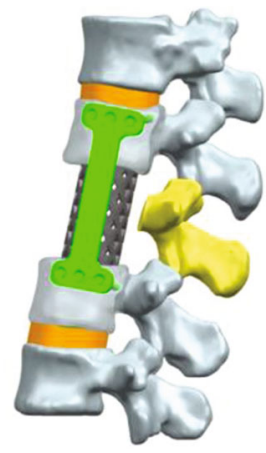

(b)

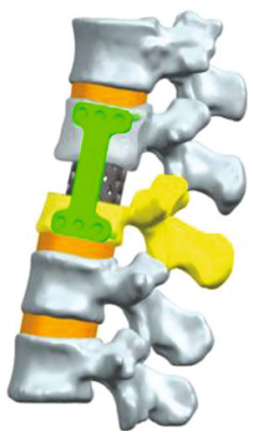

(c)

FIgURE 4: The models of different surgical protocols designed in UG NX software. (a) Anteroposterior and lateral view of the traditional double-segment screws fixation system. (b) Anteroposterior and lateral view of the novel double-segment plate fixation system. (c) Anteroposterior and lateral view of the novel single-segment plate fixation system.

internal fixation is titanium alloy, the shape of the plate is "I," and its arc is the same as that of the side-wall of the vertebral. There are 3 holes on both sides of the plate for locking screws. When inserting the locking screw, the screw should be close to the endplate and perpendicular to the sagittal axis of the vertebral body. At this time, the plate and screws can support the endplate. The screw is designed as a cortical locking screw with a diameter of $3.5 \mathrm{~mm}$-while the value of traditional screw diameter is about $5.5 \mathrm{~mm}$-and the length span is $5 \mathrm{~mm}$. The novel plate is designed in different sizes, and the width is about $26 \mathrm{~mm}$ and the length $70 \mathrm{~mm}$, for various patients to choose. Compared with the larger screw diameter in the traditional anterior system, the newly designed internal fixation apparatus reduces the screw diameter, which can complete the screw installing with only a small amount of bone remaining in the injured vertebra and achieve the goal of single-segment or double-segment fusion fixation.

2.2. Building a Normal Finite Element Model. We selected a 25-year-old male volunteer who was in good health and had never got spinal disease or pain and screened by X-ray. After explaining the risks and benefits of CT scan in detail, the volunteer signed the informed consent. On March 13, 


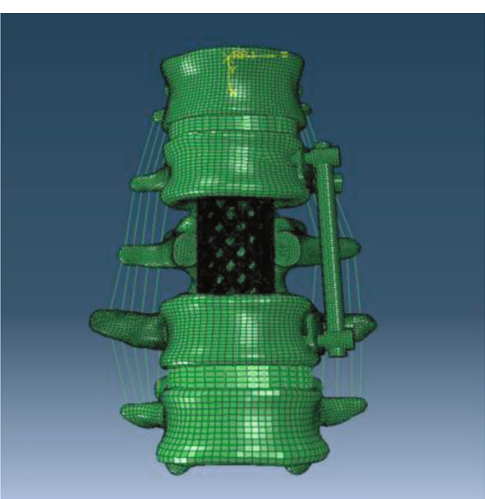

(a)

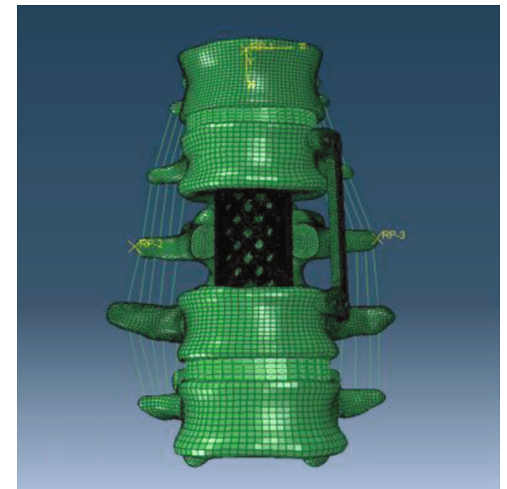

(b)

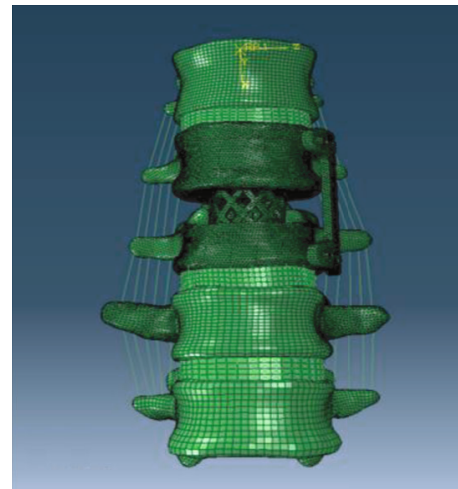

(c)

Figure 5: The models of different surgical protocols in finite element software Abaqus. Imported the meshed 3D model into the finite element software, built ligaments model, and assigned material properties. (a) Anteroposterior view of the traditional double-segment screws fixation system (model A). (b) Anteroposterior view of the novel double-segment plate fixation system (model B). (c) Anteroposterior of the novel single-segment plate fixation system (model C).

TABLE 2: Comparison between the normal spine model and models from previous studies.

\begin{tabular}{lcccc}
\hline & Results & Yamamoto et al. [16] & $\begin{array}{c}\text { ROM }\left(^{\circ} \text { ) }\right. \\
\text { Pflugmacher et al. [17] }\end{array}$ & \begin{tabular}{l} 
Basaran et al. [19] \\
\hline Flexion
\end{tabular} \\
Extension & 5.46 & $5.8 \pm 0.6$ & $5.3 \pm 1.0$ & $4.5 \pm 0.9$ \\
Left bending & 4.71 & $4.3 \pm 0.5$ & $5.7 \pm 1.0$ & $4.5 \pm 0.9$ \\
Right bending & 4.67 & $5.2 \pm 0.4$ & $4.3 \pm 0.6$ & $4.2 \pm 0.8$ \\
Left rotation & 4.55 & $4.7 \pm 0.4$ & $4.3 \pm 0.6$ & $2.3 \pm 0.6$ \\
Right rotation & 1.53 & $2.6 \pm 0.5$ & $2.1 \pm 0.5$ & $2.3 \pm 0.6$ \\
\hline
\end{tabular}

2019, the volunteer's T12-L5 vertebral was scanned by 64slice spiral CT (Siemens, Erlangen, Germany) in the CT room of the Third Hospital of Hebei Medical University. The tube current of the machine used is $200 \mathrm{~mA}$, the tube voltage is $120 \mathrm{kV}$, the slice thickness is $1 \mathrm{~mm}$, the interlayer spacing is $1 \mathrm{~mm}$, and the image data output is in DICOM (Digital Imaging and Communications in Medicine) format.

The DICOM format image data was imported into the interactive medical imaging control system Mimics17.0. The threshold segmentation was used to remove the other structures except the T12-L4 vertebral body; then, a 3D spine model of the T12 to L4 was created. These models were imported into the reverse engineering software Geomagic Studio 12.0, and the obvious defects of the vertebral body were removed using smoothing and denoising. The intervertebral disc structures of T12-L1, L1-L2, L2-L3, and L3-L4 were designed and established by using UG NX9.0 software in which the nucleus pulposus occupied $44 \%$ of the area of the intervertebral disc (Figure 2). The 3D spine model was imported into the Hypermesh program to divide the $3 \mathrm{D}$ structure into a mesh model including 441135 nodes and 975423 elements. Finally, the divided model was imported into the Abaqus finite element analysis software to establish ligament structures around the model and named M0 (Figure 3), including the anterior longitudinal ligament, posterior longitudinal ligament, ligamentum flavum, interspinous ligament, supraspinous ligament, and intertransverse ligament. Each structure was assigned material properties (Table 1) [14, 15], including elastic modulus and Poisson's ratio.

2.3. Building Anterior Depression Model of the L2. According to the characteristic of the anterior approach, the completed 3D thoracolumbar model was imported into the UG NX9.0 software to build three simulated depression models. They were divided into two forms: (1) the upper $1 / 2$ bone of the L2 vertebrae and the adjacent intervertebral disc were removed to meet the single-segment decompression and fusion surgery, and this model only created one; (2) both L2 vertebrae and all the adjacent intervertebral discs were removed to meet the double-segment decompression and fusion surgery, and two models of this type were established.

\subsection{Building Model of the Cage and Internal Fixation} Devices. The cage and internal fixation devices were built by the UG NX9.0 software (Figure 4). The material of all apparatuses was titanium alloy, and each part connected with a locked form. All the internal fixation devices were placed on the left side of the spine models. (1) The data of the traditional anterior two-segment fixation system was provided by Double-Medical Technology Co., Ltd. The diameter of the vertebral screw was $5.5 \mathrm{~mm}$, and the length was $50 \mathrm{~mm}$; the diameter of the connecting rod was $5.5 \mathrm{~mm}$, and the length was $70 \mathrm{~mm}$ and $85 \mathrm{~mm}$; the width 


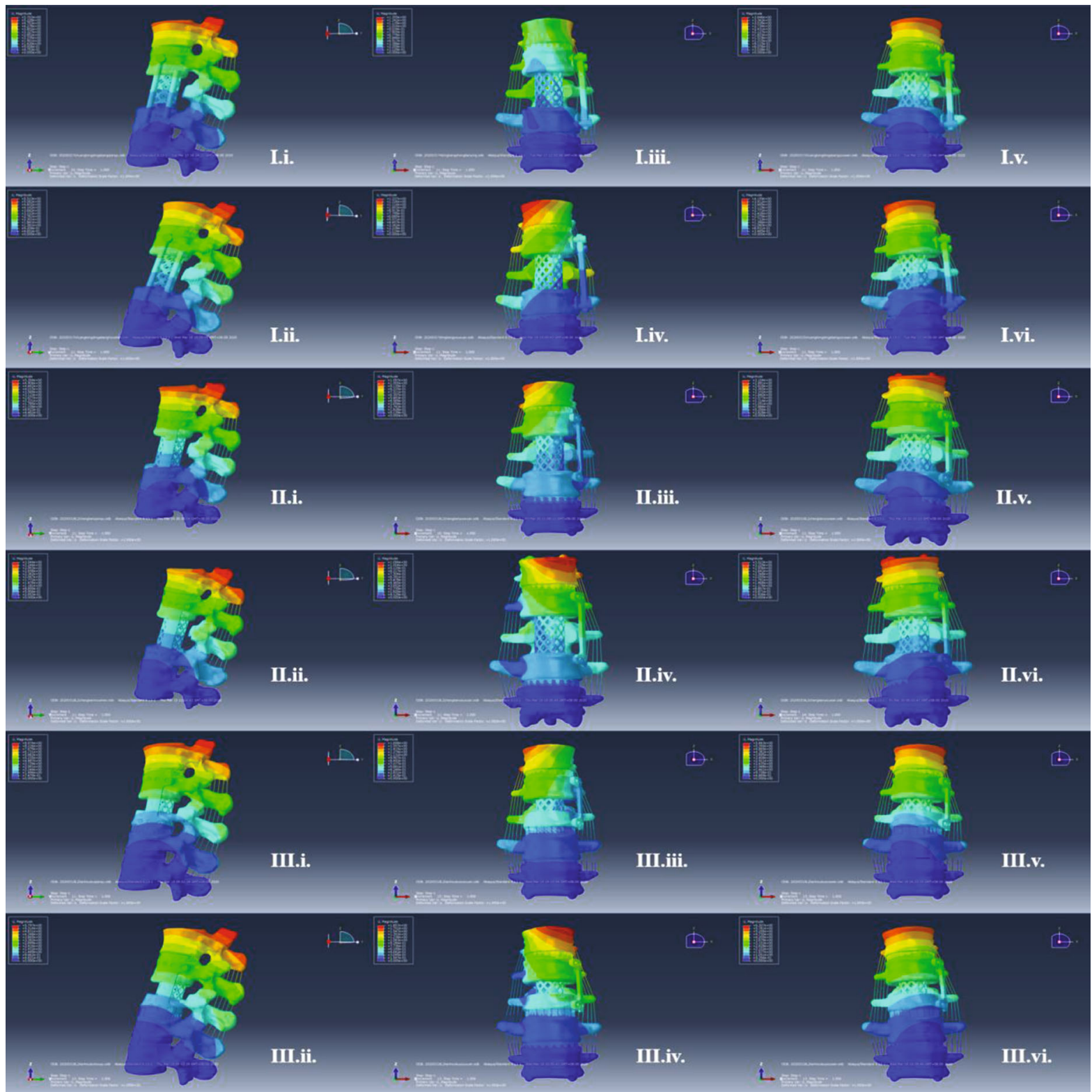

Figure 6: Movement of three models under different working conditions. Apply $500 \mathrm{~N}$ axial load and $10 \mathrm{Nm}$ torque to the endplate on T12 and observe the range of motion of the models in all directions. I.-III. represent model A, model B, and model C; i.-vi. represent different directions of motion, including flexion, extension, left axial rotation, right axial rotation, left lateral bending, and right lateral bending.

of the double hole gasket was $4 \mathrm{~mm}$, and the height was $8.5 \mathrm{~mm}$. (2) The data of the novel two-segment plate was designed by ourselves, the length of the plate was $78 \mathrm{~mm}$, and the width of the plate was $26 \mathrm{~mm}$. The screw diameter was $3.5 \mathrm{~mm}$, and the length was $45 \mathrm{~mm}$. (3) The length of the novel single-segment plate was only $20 \mathrm{~mm}$ shorter than that of the double-segment plate, while the other parameters remained unchanged. (4) Titanium cage data was provided by Double-Medical Technology Co., Ltd. In order to apply to different fusion segments, we created three cage models in two types that only had differences in length: two cages with $40 \mathrm{~mm}$ and one cage with $18 \mathrm{~mm}$. Their diameters were $24 \mathrm{~mm}$, and thicknesses were $1.5 \mathrm{~mm}$. Finally, the Hypermesh program was used to mesh all apparatuses: the traditional fixation system had 28491 nodes and 127158 elements; the novel two-segment plate had 8095 nodes and 27046 elements; the novel single-segment plate had 7773 nodes and 26400 elements; the bigger cage had 3585 nodes and 8778 elements but smaller 2230 nodes and 5957 elements.

\subsection{Establishing Different Surgical Models in Finite Element} Software. The meshed models in Hypermesh were imported into the finite element software Abaqus to give material properties (Table 1), including elastic modulus and Poisson's ratio. The two-segment fixation with the traditional instrument was named model $\mathrm{A}$, the two-segment fixation with the novel plate was named model $\mathrm{B}$, and the single-segment fixation with the novel plate was named model C (Figure 5). 


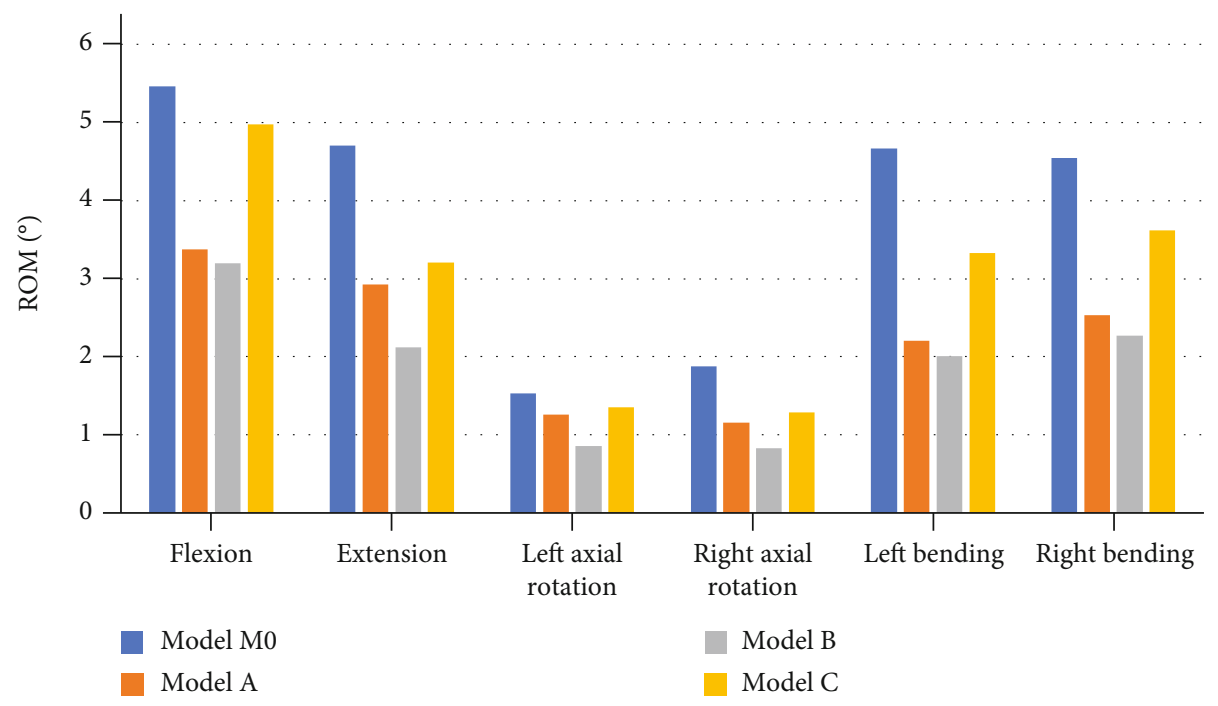

FIGURE 7: The results of range of motion.

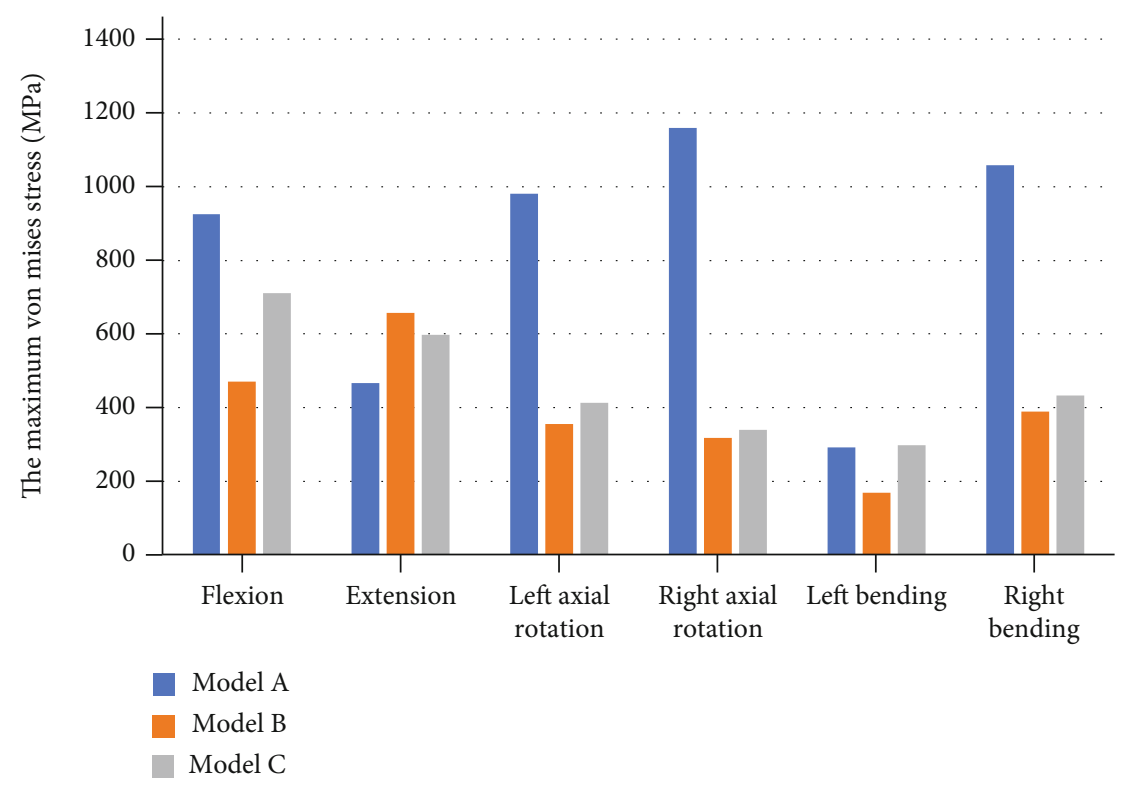

Figure 8: The maximum von Mises stress of cages.

2.6. Setting Up Loads and Boundaries. The boundaries and loads were set to simulate spinal movement using the finite element software Abaqus. The boundaries were defined as the lower part and back of the L4 vertebrae that were set to be fixed. The rotation of the spine around the $X, Y$, and $Z$ axes was defined as the flexion-extension, lateral bending, and rotation of the spine. According to normal human body weight bearing and previously published literature [16-18], an axial load of $500 \mathrm{~N}$ and a torque of $10 \mathrm{Nm}$ are uniformly applied to the T12 endplate.

2.7. Evaluation Index. It analyzed the spinal motion range of the thoracolumbar spine in 6 different directions and the maximum von Mises stress of cages and internal fixations in the three models. No statistical analysis was performed in this study as only one subject was modeled.

\section{Results}

3.1. Results of Model Validity. In order to verify the validity of the model, we applied $150 \mathrm{~N}$ axial pressure and $10 \mathrm{Nm}$ torque on the upper surface of the T12 vertebrae to measure the ROM of the model in all directions. The measured range of motion of the normal model under various working conditions is similar to the results of previous biomechanical studies (Table 2) $[16,17,19]$, which proves the validity of the finite element model established in this study.

3.2. The Results of Range of Motion. The upper surface of T12 was uniformly applied $500 \mathrm{~N}$ axial load and $10 \mathrm{Nm}$ torque to simulate spinal motion (Figure 6), and the ROM of the simulated models was recorded (Figure 7). The ROM of the three simulated models in all directions is smaller than 


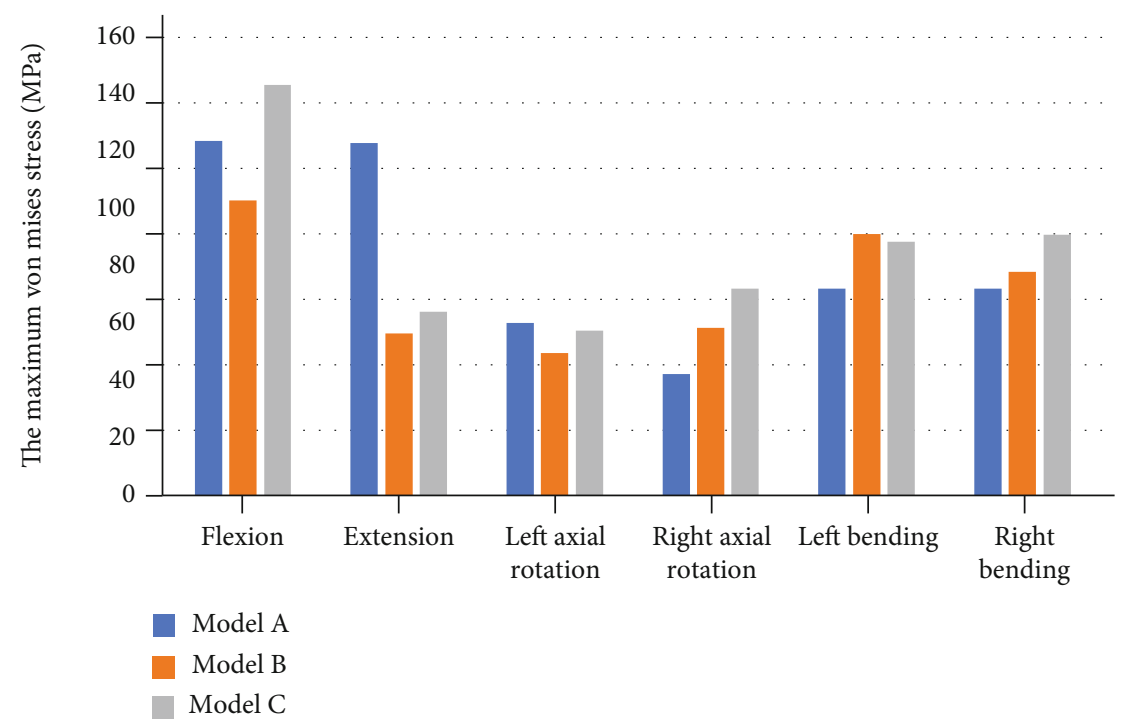

FIgURE 9: The maximum von Mises stress of internal fixations.

that of the normal model. Among the three simulated models, model $\mathrm{C}$ has the largest range of motion in six directions, and model $\mathrm{B}$ has the smallest range of motion in six directions. Compared with model B, model A has an increase of $5.63 \%$ in flexion, $38.21 \%$ in extension, $46.51 \%$ in left axial rotation, $39.76 \%$ in right axial rotation, $9.45 \%$ in left bending, and $11.45 \%$ in right bending. Compared with model B, model C has an increase of $555.63 \%$ in flexion, $51.42 \%$ in extension, $56.98 \%$ in left axial rotation, $55.42 \%$ in right axial rotation, $65.67 \%$ in left bending, and $59.47 \%$ in right bending.

3.3. The Maximum von Mises Stress of Each Internal Fixation and Cage. The maximum von Mises stress of the cages in each model in different directions of motion is shown in Figure 8. When bending on the left side, each simulated surgery model shows that the stress of the cage is the smallest. We chose model A and model B to appraise the maximum von Mises stress because they only have differences in the internal fixation device. Except in the extension direction, the stress in the other motion directions of model $\mathrm{A}$ is much greater than the stress of the cage in model B. The maximum von Mises stress of the cage in model A decreases by $29.11 \%$ in the extension but increases by $96.81 \%$ in the flexion, $175.96 \%$ in the left axial rotation, $265.73 \%$ in the right axial rotation, $73.73 \%$ in the left bending, and $171.28 \%$ in the right bending.

The maximum von Mises stress of the internal fixation devices in each model in different directions of motion is shown in Figure 9. In the flexion, extension, and left axial rotation, the maximum von Mises stress of model $\mathrm{A}$ is larger than that of the model B by $20.23 \%, 117.43 \%$, and $21.34 \%$. In the right axial rotation, left bending, and right bending, the maximum von Mises stress of model $\mathrm{A}$ is reduced by $27.38 \%, 20.77 \%$, and $7.54 \%$ compared with the model B.

\section{Discussion}

We designed a finite element experiment to compare the effects of novel plate and traditional internal fixation device on spinal stability and range of motion after surgical fixation. The results show that the novel plate provides better postfusion stability, and the reduction of fusion segments has a smaller effect on spinal mobility. Meanwhile, the new internal fixation device can better disperse the stress to avoid internal fixation stress concentration.

Anterior channel technology is mostly used in thoracolumbar degenerative diseases to treat intervertebral disc lesions and complete intervertebral fusion [20,21], but it is rarely used in thoracolumbar burst fractures. Based on the concept of minimally invasive, we use channel technology to perform anterior decompression and fusion treatment for burst fracture patients with complete PLC and neurological impairment. In the course of treatment, we found that nerve decompression can be completed with only a portion of the injured vertebrae bone removed. However, due to the cumbersome connection of the anterior screw rod internal fixation device and the relatively large screw diameter, it cannot be well matched with the channel technology to complete the fixation with little bone remaining in the injured vertebra. In view of these considerations, we newly designed a more compact and convenient device to match the working channel to achieve the purpose of obtaining maximum stability while fixing fewer segments. The "raft support" [22] concept used in the treatment of the long tubular bone metaphysis is integrated into the newly designed plate. The diameter of the screw is reduced while the number of screws is increased, so that the screws are arranged in a plane at both sides of the plate, which can support the endplates.

In this experiment, the range of motion of the three simulated models is less than that of the normal model. Previous literature has shown that no matter what kind of surgical fixation method, it will have different degrees of influence on the mobility of the spine $[23,24]$, and the results in this experiment also reflect similar problems. A comparison between model $\mathrm{A}$ and model $\mathrm{B}$ shows that only the internal fixation method is different, so the larger the movement range, the worse the stability of the internal fixation. The 
results that the novel locking plate method has a smaller ROM explain the locking manner of the novel plate, which makes the screw and the plate integrated, is simpler and more reliable than the traditional nail-rod press-fit fixing method. Spinal fusion surgery will cause changes in the pressure in the intervertebral discs of adjacent segments. Over time, the intervertebral discs of adjacent segments may undergo metamorphosis, and there is a risk of developing adjacent segment disease (ASD) [25]. Biomechanical studies of simulated lumbar fusion surgery have shown that the pressure in the adjacent intervertebral discs of the fusion segment will increase, and the increase in pressure is positively correlated with the number of fusion segments $[26,27]$. The longer the fusion segment, the more likely it is to develop ASD. The newly designed plate reduces the diameter of the screw which requires less residual bone in the injured vertebra so it might reduce the fusion segment to decrease the incidence of ASD.

We also measured the maximum von Mises stress of each internal fixation and cage to judge the support effect of different internal fixation devices on the spine. The cage is located between the two vertebral bodies, and its force can reflect the amount of stress shared by the internal fixation. Our results of the maximum stress of the cage indicate that the new plate bears more stress than the traditional device, because the screws are arranged in a plane to provide planar support to the endplate, which provides stronger stability than the traditional linear support structure with two nails. Maintaining a stable state can also provide a better mechanical environment for the growth and fusion of bone tissue. Although the new plate bears more stress, the maximum stress value on the internal fixation is smaller than that of the nail-rod system in the movement direction of flexion, extension, and left axial rotation. In the new plate system, the screw and the plate form a whole through a locking structure, which disperses the stress on the screw-plate structure and is not prone to stress concentration, thereby reducing fatigue break of the internal fixation.

There are also some shortcomings in the research. Although the finite element software can simulate movement of spine and measure the stress on the internal fixation, it only loads the force in six directions and still cannot fully simulate all the characteristics of the human body in which the movement of the spine is produced by the contraction of different muscles. Various material properties are also assigned with reference to different documents, which may be different from the real organization. The method of finite element analysis will have personal errors when the model is established, and some structures will be different from the real spine condition. Moreover, maximum stress experiments and fatigue experiments are required to further test the properties of the plate. If necessary, we will further improve the device because the new plate is still in the experimental stage.

\section{Abbreviations}

PLC: $\quad$ Posterior ligamentous complex

TDFI: The traditional double-segment fixation instrument

NSFI: The novel instrument in single-segment fixation instrument
NDFI: The novel instrument in double-segment fixation instrument

ROM: Range of motion

3D: $\quad$ Three dimensional

CT: $\quad$ Computed tomography

DICOM: Digital imaging and communications in medicine ASD: $\quad$ Adjacent segment disease.

\section{Data Availability}

All data analyzed during this study are included in this article.

\section{Ethical Approval}

This article does not contain any studies with human participants or animals performed by any of the authors. We confirm that all methods were performed in accordance with the relevant guidelines and regulations.

\section{Consent}

Not applicable.

\section{Conflicts of Interest}

The authors declare that they have no competing interests.

\section{Authors' Contributions}

All authors contributed to the study conception and design. Material preparation, data collection, and analysis were performed by Chongyao Lu, Xiaodong Cheng, and Xiaodong Bai. The first draft of the manuscript was written by Pengcheng Ren, and all authors commented on previous versions of the manuscript. All authors read and approved the final manuscript.

\section{Acknowledgments}

We would like to thank Yanbin Zhu for his assistance in the conduction of finite element analysis.

\section{References}

[1] K. Alpantaki, A. Bano, D. Pasku et al., "Thoracolumbar burst fractures: a systematic review of management," Orthopedics, vol. 33, no. 6, pp. 422-429, 2010.

[2] A. R. Vaccaro, R. A. Lehman, R. J. Hurlbert et al., "A new classification of thoracolumbar injuries: the importance of injury morphology, the integrity of the posterior ligamentous complex, and neurologic status," Spine, vol. 30 , no. 20, pp. 2325 2333, 2005.

[3] J. Middendorp, A. A. Patel, and M. Schuetz, "The precision, accuracy and validity of detecting posterior ligamentous complex injuries of the thoracic and lumbar spine: a critical appraisal of the literature," European Spine Journal, vol. 22, no. 3, 2012.

[4] Y. Shono, P. C. Mcafee, and B. W. Cunningham, "Experimental study of thoracolumbar burst fractures. A radiographic and 
biomechanical analysis of anterior and posterior instrumentation systems," Spine, vol. 19, no. 15, pp. 1711-1722, 1994.

[5] T. Faciszewski, R. B. Winter, J. E. Lonstein, F. Denis, and L. Johnson, "The surgical and medical perioperative complications of anterior spinal fusion surgery in the thoracic and lumbar spine in adults. A review of 1223 procedures," Spine, vol. 20, no. 14, pp. 1592-1599, 1995.

[6] M. Mcdonnell, "Perioperative complications of anterior procedures on the spine," The Journal of Bone and Joint Surgery. American Volume, vol. 78, pp. 839-847, 1996.

[7] R. J. Oskouian and J. P. Johnson, "Vascular complications in anterior thoracolumbar spinal reconstruction," Journal of Neurosurgery, vol. 96, 1 Suppl, pp. 1-5, 2002.

[8] S. S. Kulkarni, G. L. Lowery, and R. E. Ross, "Arterial complications following anterior lumbar interbody fusion: report of eight cases," European Spine Journal, vol. 12, pp. 48-54, 2003.

[9] L. A. Snyder, J. O'Toole, K. M. Eichholz, M. J. Perez-Cruet, and R. Fessler, "The technological development of minimally invasive spine surgery," Journal of Biomedicine and Biotechnology, vol. 2014, Article ID 293582, 9 pages, 2014.

[10] J. Park, D. W. Ham, B. T. Kwon, S. M. Park, H. J. Kim, and J. S. Yeom, "Minimally invasive spine surgery: techniques, technologies, and indications," Asian Spine Journal, vol. 14, no. 5, pp. 694-701, 2020.

[11] D. S. Xu, C. T. Walker, G. Jakub, J. D. Turner, S. William, and J. S. Uribe, "Minimally invasive anterior, lateral, and oblique lumbar interbody fusion: a literature review," Annals of Translational Medicine, vol. 6, no. 6, p. 104, 2018.

[12] R. Li, X. Li, H. Zhou, and W. Jiang, "Development and application of oblique lumbar interbody fusion," Orthopaedic Surgery, vol. 12, no. 2, pp. 355-365, 2020.

[13] W. D. Smith, E. Dakwar, T. V. le, G. Christian, S. Serrano, and J. S. Uribe, "Minimally invasive surgery for traumatic spinal Pathologies," Spine (Phila Pa 1976), vol. 35, Supplement, pp. S338-S346, 2010.

[14] Y. Kim and T. W. Kim, "Finite element analysis of the effects of pedicle screw fixation nut loosening on lumbar interbody fusion based on the elasto-plateau plasticity of bone characteristics," Spine, vol. 35, no. 6, pp. 599-606, 2010.

[15] G. Denozière and D. N. Ku, "Biomechanical comparison between fusion of two vertebrae and implantation of an artificial intervertebral disc," Journal of Biomechanics, vol. 39, no. 4, pp. 766-775, 2006.

[16] I. Yamamoto, M. M. Panjabi, T. Crisco, and T. Oxland, "Three-dimensional movements of the whole lumbar spine and lumbosacral joint," Spine, vol. 14, no. 11, pp. 1256-1260, 1989.

[17] R. Pflugmacher, P. Schleicher, J. Schaefer et al., "Biomechanical comparison of expandable cages for vertebral body replacement in the thoracolumbar spine," Spine, vol. 29, no. 13, pp. 1413-1419, 2004.

[18] C. Li, Z. Yue, H. Wang, J. Liu, L. Xiang, and S. Mohammed, "Treatment of unstable thoracolumbar fractures through short segment pedicle screw fixation techniques using pedicle fixation at the level of the fracture: a finite element analysis," PLoS One, vol. 9, no. 6, article e99156, 2014.

[19] R. Basaran, M. Efendioglu, M. Kaksi, T. Celik, İ. Mutlu, and M. Ucar, "Finite element analysis of short- versus longsegment posterior fixation for thoracolumbar burst fracture," World Neurosurgery, vol. 128, pp. e1109-e1117, 2019.
[20] A. Yong, M. S. Youn, and H. H. Dong, "Endoscopic transforaminal lumbar interbody fusion: a comprehensive review," Expert Review of Medical Devices, vol. 16, no. 2, pp. 1-8, 2019.

[21] Z. Chen, L. Zhang, J. Dong et al., "Percutaneous transforaminal endoscopic discectomy compared with microendoscopic discectomy for lumbar disc herniation: 1-year results of an ongoing randomized controlled trial," Journal of Neurosurgery. Spine, vol. 28, no. 3, pp. 300-310, 2018.

[22] H. Sun, Y. Zhu, Q. F. He, L. Y. Shu, W. Zhang, and Y. M. Chai, "Reinforcement strategy for lateral rafting plate fixation in posterolateral column fractures of the tibial plateau: the magic screw technique," Injury, vol. 48, no. 12, pp. 2814-2826, 2017.

[23] M. Gehrchen, S. K. Hegde, M. Moldavsky et al., "Range of motion after thoracolumbar corpectomy: evaluation of analogous constructs with a novel low-profile anterior dual-rod system and a traditional dual-rod system," European Spine Journal, vol. 26, no. 3, pp. 666-670, 2017.

[24] M. F. Metzger, S. T. Robinson, R. C. Maldonado, J. Rawlinson, J. Liu, and F. L. Acosta, "Biomechanical analysis of lateral interbody fusion strategies for adjacent segment degeneration in the lumbar spine," The Spine Journal, vol. 17, no. 7, pp. 1004-1011, 2017.

[25] C. Scemama, B. Magrino, P. Gillet, and P. Guigui, "Risk of adjacent-segment disease requiring surgery after short lumbar fusion: results of the french spine surgery society series," Journal of Neurosurgery. Spine, vol. 25, no. 1, pp. 46-51, 2016.

[26] H. M. Robbins, "Adjacent segment degeneration and adjacent segment disease: the consequences of spinal fusion?," The Spine Journal, vol. 4, no. 6, pp. S190-S194, 2004.

[27] Q. T. Zhang, L. C. Wang, and M. Yao, "The biologic mechanisms for intervertebral disc degeneration (Chin)," Zhonghua Gu Ke Za Zhi, vol. 26, pp. 206-210, 2006. 